\title{
Cobalt-free Nickel-rich positive electrode materials with a core-shell structure
}

Ning Zhang,,${ }^{\dagger}+$ Nafiseh Zaker, $"$ Hongyang Li,,${ }^{\ddagger}$ Aaron Liu, ${ }^{\S}$ Julie Inglis, ${ }^{\perp}$ Linda Jing, ${ }^{\#}$ Jing Li, ${ }^{\#}$ Ying Li, ${ }^{\dagger}$ Gianluigi A. Botton, $"$ and Jeff R. Dahn ${ }^{z, \star}, \S$

†School of Metallurgy, Northeastern University, Shenyang 110819, China,

" Materials Science and Engineering, Canadian Centre for Electron Microscopy, McMaster University, Hamilton, Ontario L8S 4L7, Canada,

Dept. of Physics and Atmosphere Science, Dalhousie University, Halifax, Nova Scotia B3H 3J5, Canada,

§Dept. of Chemistry, Dalhousie University, Halifax, Nova Scotia B3H 4R2, Canada,

${ }^{\perp}$ McMaster University, Hamilton, ON L8S 4L8, Canada

${ }^{\#}$ Tesla, Palo Alto, California 94304, USA

${ }^{z}$ Corresponding authors-jeff.dahn@dal.ca 
Table S1. Summary table of Rietveld refinement results of samples with different particle size, different Li/TM ratio, and different synthesis temperature.

\begin{tabular}{|c|c|c|c|c|c|c|c|c|c|}
\hline$\#$ & Sample ID & $\begin{array}{l}\text { Particle size } \\
(\mu \mathrm{m})\end{array}$ & $\begin{array}{l}\text { Core: shell } \\
\text { ratio }\end{array}$ & $\begin{array}{l}\text { Synthesis } \\
\text { temperature }\left({ }^{\circ} \mathrm{C}\right)\end{array}$ & Li/TM ratio & $a( \pm 0.0001 \AA ̊)$ & $c( \pm 0.0005 \AA ̊)$ & $\begin{array}{l}\mathrm{Ni} \text { in } \mathrm{Li} \\
\text { layer\%( } \pm 0.3)\end{array}$ & Bragg-f \\
\hline 7 & $\mathrm{LiNi}_{0.95} \mathrm{Al}_{0.05} \mathrm{O}_{2}$ & 10 & $\mathrm{~N} / \mathrm{A}$ & 700 & 1.02 & 2.8737 & 14.2075 & 2.0 & 1.57 \\
\hline 8 & $\mathrm{LiNi}_{0.95} \mathrm{Al}_{0.05} \mathrm{O}_{2}$ & 18 & $\mathrm{~N} / \mathrm{A}$ & 700 & 1.02 & 2.8703 & 14.2019 & 1.2 & 1.49 \\
\hline 9 & $\begin{array}{l}\text { CS- } \\
\text { LNO:NiMn83/17 }\end{array}$ & 10 & 2.5 & 700 & 0.98 & 2.8787 & 14.2009 & 5.8 & 2.67 \\
\hline 10 & $\begin{array}{l}\text { CS- } \\
\text { LNO:NiMn83/17 }\end{array}$ & 10 & 2.5 & 700 & 1.00 & 2.8785 & 14.2021 & 5.9 & 2.91 \\
\hline 11 & $\begin{array}{l}\text { CS- } \\
\text { LNO:NiMn83/17 }\end{array}$ & 10 & 2.5 & 700 & 1.02 & 2.8777 & 14.2000 & 5.6 & 2.97 \\
\hline 12 & $\mathrm{LiNi}_{0.95} \mathrm{Mn}_{0.05} \mathrm{O}_{2}$ & 10 & $\mathrm{~N} / \mathrm{A}$ & 700 & 1.00 & 2.8759 & 14.1879 & 6.2 & 4.02 \\
\hline 13 & $\mathrm{LiNi}_{0.95} \mathrm{Mn}_{0.05} \mathrm{O}_{2}$ & 10 & $\mathrm{~N} / \mathrm{A}$ & 700 & 1.02 & 2.8778 & 14.1999 & 5.8 & 3.79 \\
\hline 14 & $\mathrm{LiNi}_{0.95} \mathrm{Mn}_{0.05} \mathrm{O}_{2}$ & 10 & $\mathrm{~N} / \mathrm{A}$ & 700 & 1.04 & 2.8739 & 14.1866 & 5.1 & 3.40 \\
\hline 15 & $\mathrm{LiNi}_{0.95} \mathrm{Mn}_{0.05} \mathrm{O}_{2}$ & 10 & $\mathrm{~N} / \mathrm{A}$ & 700 & 1.08 & 2.8715 & 14.1819 & 5.4 & 2.80 \\
\hline 16 & $\mathrm{LiNi}_{0.95} \mathrm{Mn}_{0.05} \mathrm{O}_{2}$ & 10 & $\mathrm{~N} / \mathrm{A}$ & 700 & 1.12 & 2.8717 & 14.1784 & 6.0 & 3.87 \\
\hline 17 & $\mathrm{LiNi}_{0.95} \mathrm{Mn}_{0.05} \mathrm{O}_{2}$ & 10 & $\mathrm{~N} / \mathrm{A}$ & 735 & 1.00 & 2.8757 & 14.1972 & 3.7 & 2.23 \\
\hline 18 & $\mathrm{LiNi}_{0.95} \mathrm{Mn}_{0.05} \mathrm{O}_{2}$ & 10 & $\mathrm{~N} / \mathrm{A}$ & 735 & 1.08 & 2.8753 & 14.1978 & 2.9 & 1.71 \\
\hline 19 & $\mathrm{LiNi}_{0.95} \mathrm{Mn}_{0.05} \mathrm{O}_{2}$ & 10 & $\mathrm{~N} / \mathrm{A}$ & 735 & 1.12 & 2.8749 & 14.1948 & 2.9 & 2.12 \\
\hline 20 & $\mathrm{LiNi}_{0.95} \mathrm{Mn}_{0.05} \mathrm{O}_{2}$ & 10 & $\mathrm{~N} / \mathrm{A}$ & 735 & 1.17 & 2.875 & 14.1955 & 2.4 & 1.63 \\
\hline
\end{tabular}


Table S2. (003), (101), (104) and (018) Full width at half maximum (FWHM, in 20) and peak position data from Rietveld refinement results.

\begin{tabular}{|c|c|c|c|c|c|c|c|c|c|c|c|}
\hline$\#$ & Sample ID & $\begin{array}{l}\text { Particle } \\
\text { size } \\
(\mu \mathrm{m})\end{array}$ & $\begin{array}{l}\text { Temps } \\
\left({ }^{\circ} \mathrm{C}\right)\end{array}$ & $\begin{array}{l}\text { FWHM } 2 \theta \\
\left({ }^{\circ}\right)(003)\end{array}$ & $\begin{array}{l}\text { Peak } \\
\text { position }\left({ }^{\circ}\right) \\
(003)\end{array}$ & $\begin{array}{l}\text { FWHM } 2 \theta \\
\left(^{\circ}\right)(101)\end{array}$ & $\begin{array}{l}\text { Peak } \\
\text { position }\left({ }^{\circ}\right) \\
(101)\end{array}$ & $\begin{array}{l}\text { FWHM } 2 \theta \\
\left({ }^{\circ}\right)(104)\end{array}$ & $\begin{array}{l}\text { Peak } \\
\left.\text { position ( }{ }^{\circ}\right) \\
(104)\end{array}$ & $\begin{array}{l}\text { FWHM } 2 \theta \\
\left({ }^{\circ}\right)(018)\end{array}$ & $\begin{array}{l}\text { Peak } \\
\text { position }\left({ }^{\circ}\right) \\
(018)\end{array}$ \\
\hline 1 & $\begin{array}{l}\text { CS- } \\
\text { LNO:NiMg83/17 }\end{array}$ & 10 & 700 & 0.100 & 18.706 & 0.137 & 36.542 & 0.161 & 44.325 & 0.238 & 64.30 \\
\hline 2 & $\mathrm{LiNi}_{0.95} \mathrm{Mg}_{0.05} \mathrm{O}_{2}$ & 210 & 700 & 0.084 & 18.711 & 0.127 & 36.579 & 0.147 & 44.361 & 0.207 & 64.338 \\
\hline 3 & $\begin{array}{l}\text { CS- } \\
\text { LNO:NiAl83/17 }\end{array}$ & 5 & 700 & 0.140 & 18.723 & 0.194 & 36.641 & 0.224 & 44.424 & 0.319 & 64.409 \\
\hline 4 & $\mathrm{LiNi}_{0.95} \mathrm{Al}_{0.05} \mathrm{O}_{2}$ & 3 & 700 & 0.095 & 18.722 & 0.136 & 36.629 & 0.155 & 44.412 & 0.214 & 64.397 \\
\hline 5 & $\begin{array}{l}\text { CS- } \\
\text { LNO:NiMn83/17 }\end{array}$ & 10 & 700 & 0.175 & 18.731 & 0.213 & 36.578 & 0.238 & 44.376 & 0.320 & 64.387 \\
\hline 6 & $\mathrm{LiNi}_{0.95} \mathrm{Mn}_{0.05} \mathrm{O}_{2}$ & ${ }_{2} 10$ & 735 & 0.097 & 18.737 & 0.134 & 36.625 & 0.152 & 44.420 & 0.203 & 64.433 \\
\hline 7 & $\mathrm{LiNi}_{0.95} \mathrm{Al}_{0.05} \mathrm{O}_{2}$ & 10 & 700 & 0.101 & 18.721 & 0.144 & 36.626 & 0.164 & 44.409 & 0.221 & 64.393 \\
\hline 18 & $\mathrm{LiNi}_{0.95} \mathrm{Mn}_{0.05} \mathrm{O}_{2}$ & & 700 & 0.132 & 18.749 & 0.166 & 36.626 & 0.184 & 44.430 & 0.242 & 64.462 \\
\hline
\end{tabular}


Table S3. Summary table of half-cell data at $\mathrm{C} / 20$ and $\mathrm{C} / 5$ at $30^{\circ} \mathrm{C}, 3-4.3 \mathrm{~V}$ vs. $\mathrm{Li} / \mathrm{Li}^{+}$.

\begin{tabular}{|c|c|c|c|c|c|c|c|c|c|c|}
\hline$\#$ & Sample ID & $\begin{array}{l}\text { Particle } \\
\text { size }(\mu \mathrm{m})\end{array}$ & $\begin{array}{l}\text { Core: shell } \\
\text { ratio }\end{array}$ & $\begin{array}{l}\text { Synthesis } \\
\text { temperature }\left({ }^{\circ} \mathrm{C}\right)\end{array}$ & $\begin{array}{l}\text { Li/TM } \\
\text { ratio }\end{array}$ & $\begin{array}{l}1^{\text {st }} \mathrm{C} / 20 \mathrm{CC} \\
(\mathrm{mAh} / \mathrm{g})\end{array}$ & $\begin{array}{l}1^{\text {st }} \mathrm{C} / 20 \mathrm{DC} \\
(\mathrm{mAh} / \mathrm{g})\end{array}$ & $1^{\text {st }}$ IRC $\%$ & $\begin{array}{l}1^{\mathrm{st}} \mathrm{C} / 5 \mathrm{DC} \\
(\mathrm{mAh} / \mathrm{g})\end{array}$ & $\begin{array}{l}\text { Cap. Retention } \\
\text { after } 50 \text { cycles } \\
\text { at } \mathrm{C} / 5\end{array}$ \\
\hline 1 & $\begin{array}{l}\text { CS- } \\
\text { LNO:NiMg83/17 }\end{array}$ & 710 & 2.5 & 700 & 1.02 & $225.6(4)$ & $193.6(1)$ & $14.2(2)$ & $177(2)$ & $0.955(9)$ \\
\hline 2 & $\mathrm{LiNi}_{0.95} \mathrm{Mg}_{0.05} \mathrm{O}_{2}$ & 10 & N/A & 700 & 1.02 & $226.7(3)$ & 194(1) & $14.3(4)$ & $184.5(5)$ & $0.98(1)$ \\
\hline 3 & $\begin{array}{l}\text { CS- } \\
\text { LNO:NiAl83/17 }\end{array}$ & 5 & 2.5 & 700 & 1.02 & $241.4(3)$ & $228.3(4)$ & $5.4(1)$ & $222(2)$ & $0.925(7)$ \\
\hline 4 & $\mathrm{LiNi}_{0.95} \mathrm{Al}_{0.05} \mathrm{O}_{2}$ & 3 & N/A & 700 & 1.02 & $244(1)$ & $229.1(7)$ & $6.2(2)$ & 210.0(9) & $0.813(7)$ \\
\hline 5 & $\begin{array}{l}\text { CS- } \\
\text { LNO:NiMn83/17 }\end{array}$ & 710 & 2.5 & 700 & 1.04 & $235.2(5)$ & $212.2(2)$ & $9.8(1)$ & 209.5(8) & $0.953(8)$ \\
\hline 6 & $\mathrm{LiNi}_{0.95} \mathrm{Mn}_{0.05} \mathrm{O}_{2}$ & 10 & N/A & 735 & 1.04 & 231.8(9) & 212.5(9) & $8.3(0)$ & 204.9(4) & $0.949(6)$ \\
\hline 7 & $\mathrm{LiNi}_{0.95} \mathrm{Al}_{0.05} \mathrm{O}_{2}$ & 10 & N/A & 700 & 1.02 & 240.5 & 218.7 & 9.1 & 206.3 & 0.934 \\
\hline 8 & $\mathrm{LiNi}_{0.95} \mathrm{Al}_{0.05} \mathrm{O}_{2}$ & 18 & N/A & 700 & 1.02 & $244.6(8)$ & $218.7(9)$ & $10.6(1)$ & $\mathrm{N} / \mathrm{A}$ & N/A \\
\hline 9 & $\begin{array}{l}\text { CS- } \\
\text { LNO:NiMn83/17 }\end{array}$ & $7^{10}$ & 2.5 & 700 & 0.98 & $234(2)$ & 205(4) & $12(1)$ & 193(3) & $0.98(2)$ \\
\hline 10 & $\begin{array}{l}\text { CS- } \\
\text { LNO:NiMn83/17 }\end{array}$ & $7^{10}$ & 2.5 & 700 & 1.00 & $239(2)$ & $215.9(1)$ & $9.6(6)$ & $207.3(2)$ & $0.921(0)$ \\
\hline 11 & $\begin{array}{l}\text { CS- } \\
\text { LNO:NiMn83/17 }\end{array}$ & $7^{10}$ & 2.5 & 700 & 1.02 & $234.8(5)$ & $207(3)$ & $11.8(9)$ & 202.3(0) & $0.955(3)$ \\
\hline 12 & $\mathrm{LiNi}_{0.95} \mathrm{Mn}_{0.05} \mathrm{O}_{2}$ & 10 & N/A & 700 & 1.00 & $224(3)$ & $196(5)$ & $13(1)$ & $174(4)$ & $0.963(0)$ \\
\hline 13 & $\mathrm{LiNi}_{0.95} \mathrm{Mn}_{0.05} \mathrm{O}_{2}$ & 10 & N/A & 700 & 1.02 & $236(4)$ & $214(3)$ & $9.3(6)$ & N/A & $\mathrm{N} / \mathrm{A}$ \\
\hline 14 & $\mathrm{LiNi}_{0.95} \mathrm{Mn}_{0.05} \mathrm{O}_{2}$ & 10 & $\mathrm{~N} / \mathrm{A}$ & 700 & 1.04 & 218.3 & 188.4 & 13.7 & 171.7 & 1.033 \\
\hline 15 & $\mathrm{LiNi}_{0.95} \mathrm{Mn}_{0.05} \mathrm{O}_{2}$ & 10 & N/A & 700 & 1.08 & 219.7 & 186.9 & 14.9 & 168.7 & 0.990 \\
\hline 16 & $\mathrm{LiNi}_{0.95} \mathrm{Mn}_{0.05} \mathrm{O}_{2}$ & 10 & N/A & 700 & 1.12 & 223.8 & 191.2 & 14.6 & 170.2 & 1.016 \\
\hline 17 & $\mathrm{LiNi}_{0.95} \mathrm{Mn}_{0.05} \mathrm{O}_{2}$ & 10 & N/A & 735 & 1.00 & $239(2)$ & $220.4(9)$ & $7.6(4)$ & $209(2)$ & $0.907(5)$ \\
\hline 18 & $\mathrm{LiNi}_{0.95} \mathrm{Mn}_{0.05} \mathrm{O}_{2}$ & 10 & $\mathrm{~N} / \mathrm{A}$ & 735 & 1.08 & $234.9(2)$ & 221.4(9) & $5.8(3)$ & $208.0(2)$ & $0.888(8)$ \\
\hline 19 & $\mathrm{LiNi}_{0.95} \mathrm{Mn}_{0.05} \mathrm{O}_{2}$ & 10 & N/A & 735 & 1.12 & $233.3(3)$ & $221.1(7)$ & $5.2(2)$ & $206.1(1)$ & $0.80(3)$ \\
\hline 20 & $\mathrm{LiNi}_{0.95} \mathrm{Mn}_{0.05} \mathrm{O}_{2}$ & 10 & N/A & 735 & 1.17 & $225.7(7)$ & $212.9(9)$ & $5.6(1)$ & 199(1) & $0.80(2)$ \\
\hline
\end{tabular}


Figure S1. XRD patterns of a) $\mathrm{Ni}_{1-\mathrm{x}} \mathrm{Mg}_{\mathrm{x}}(\mathrm{OH})_{2}(\mathrm{x}=0,0.05$, and 0.1$)$ and $\left.\mathrm{b}\right) \mathrm{Ni}_{1-\mathrm{x}} \mathrm{Mn}_{\mathrm{x}}(\mathrm{OH})_{2}(\mathrm{x}=0$, $0.05,0.1,0.15$, and 0.2 ).

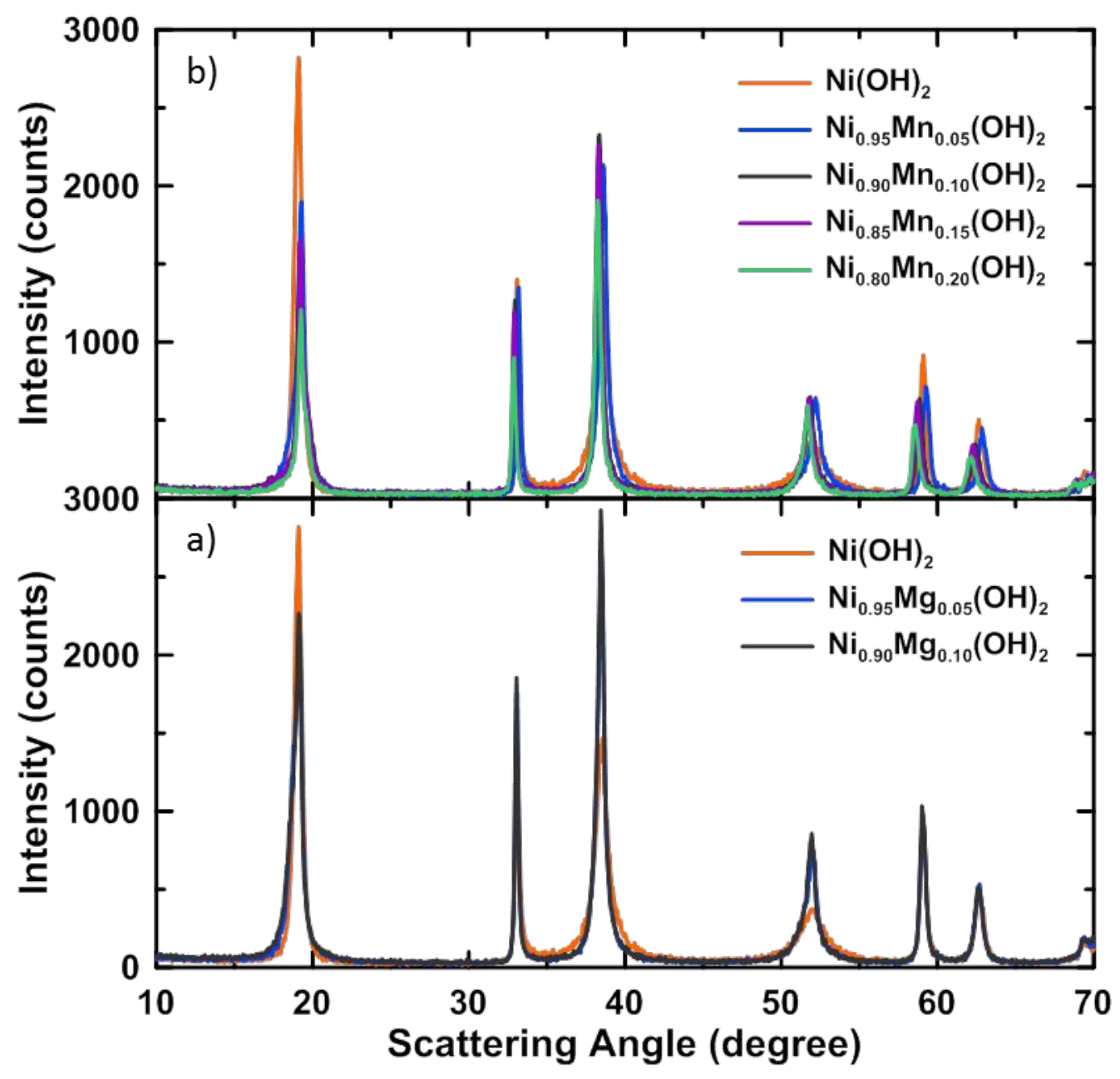


Figure S2. SEM images of a) CS-Ni(OH $)_{2}: \mathrm{Ni}_{0.83} \mathrm{Mg}_{0.17}(\mathrm{OH})_{2}$, b) CS-Ni(OH) $2: \mathrm{Ni}_{0.83} \mathrm{Al}_{0.17}(\mathrm{OH})_{2}$, and c) $\mathrm{CS}-\mathrm{Ni}(\mathrm{OH})_{2}: \mathrm{Ni}_{0.83} \mathrm{Mn}_{0.17}(\mathrm{OH})_{2}$.

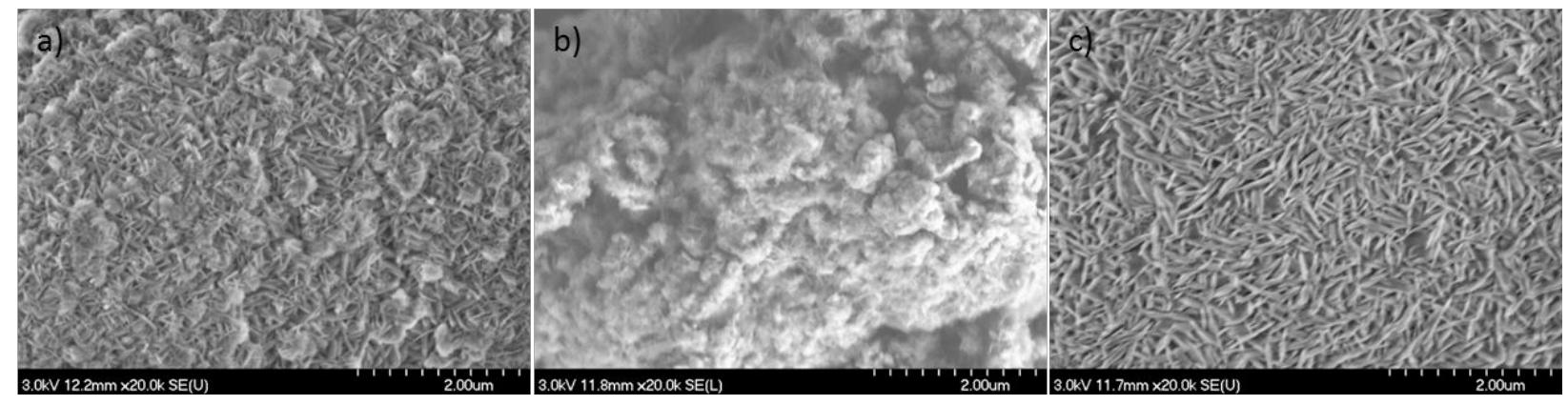


Figure S3. Cross-sectional SEM images of CS-Ni(OH $)_{2}: \mathrm{Ni}_{0.83} \mathrm{Mg}_{0.17}(\mathrm{OH})_{2}$ in a) back scattering electron mode, $b$ ) secondary electron mode; cross-sectional SEM images of CS$\mathrm{Ni}(\mathrm{OH})_{2}: \mathrm{Ni}_{0.83} \mathrm{Al}_{0.17}(\mathrm{OH})_{2}$ in c) back scattering electron mode, d) secondary electron mode; cross-sectional SEM images of CS-Ni(OH) $: \mathrm{Ni}_{0.83} \mathrm{Mn}_{0.17}(\mathrm{OH})_{2}$ in e) back scattering electron mode, f) secondary electron mode.

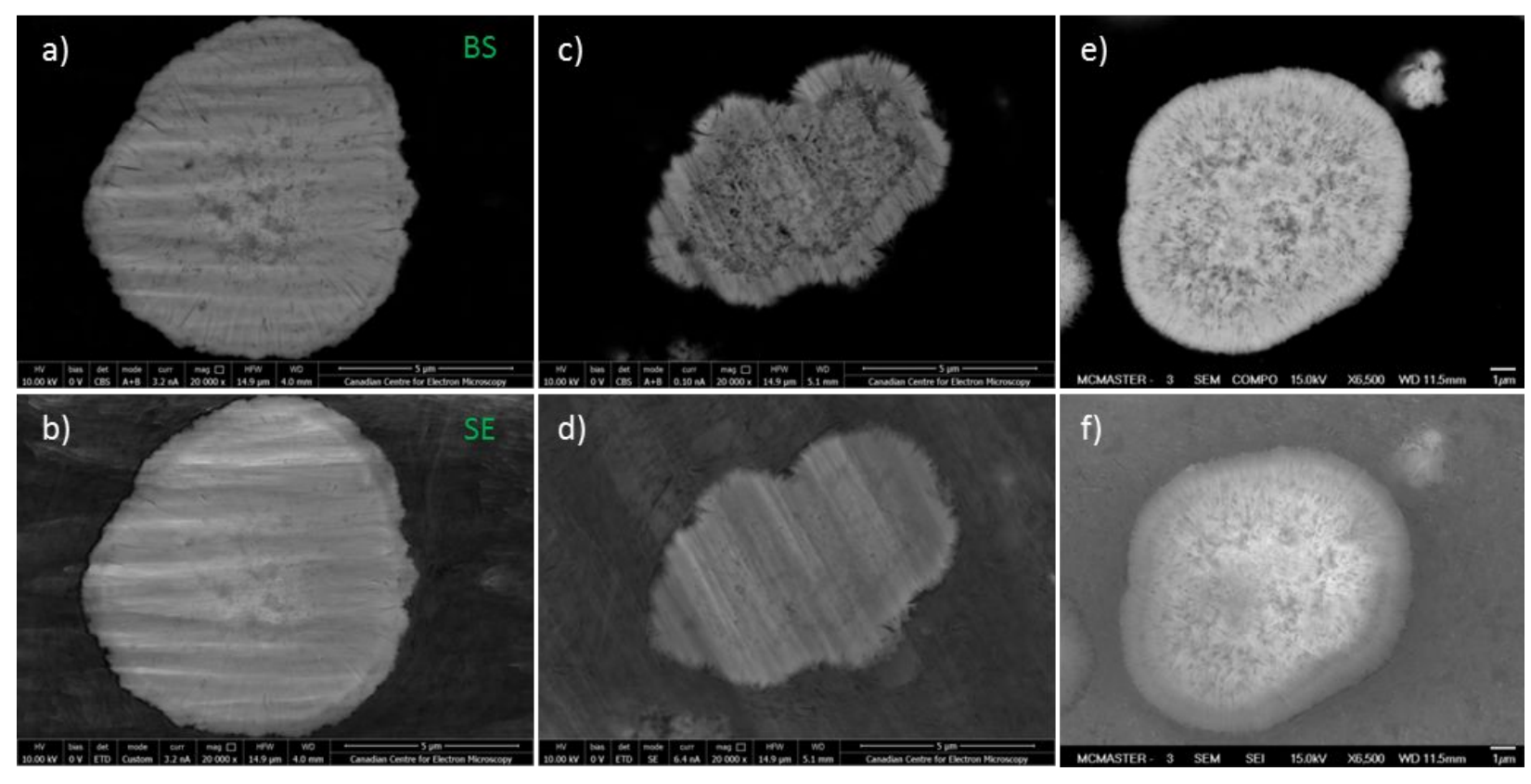


Figure S4. Particle size distribution of CS-Ni(OH) $)_{2}: \mathrm{Ni}_{0.83} \mathrm{Mg}_{0.17}(\mathrm{OH})_{2}$, CS-

$\mathrm{Ni}(\mathrm{OH})_{2}: \mathrm{Ni}_{0.83} \mathrm{Al}_{0.17}(\mathrm{OH})_{2}$, and $\mathrm{CS}-\mathrm{Ni}(\mathrm{OH})_{2}: \mathrm{Ni}_{0.83} \mathrm{Mn}_{0.17}(\mathrm{OH})_{2}$.

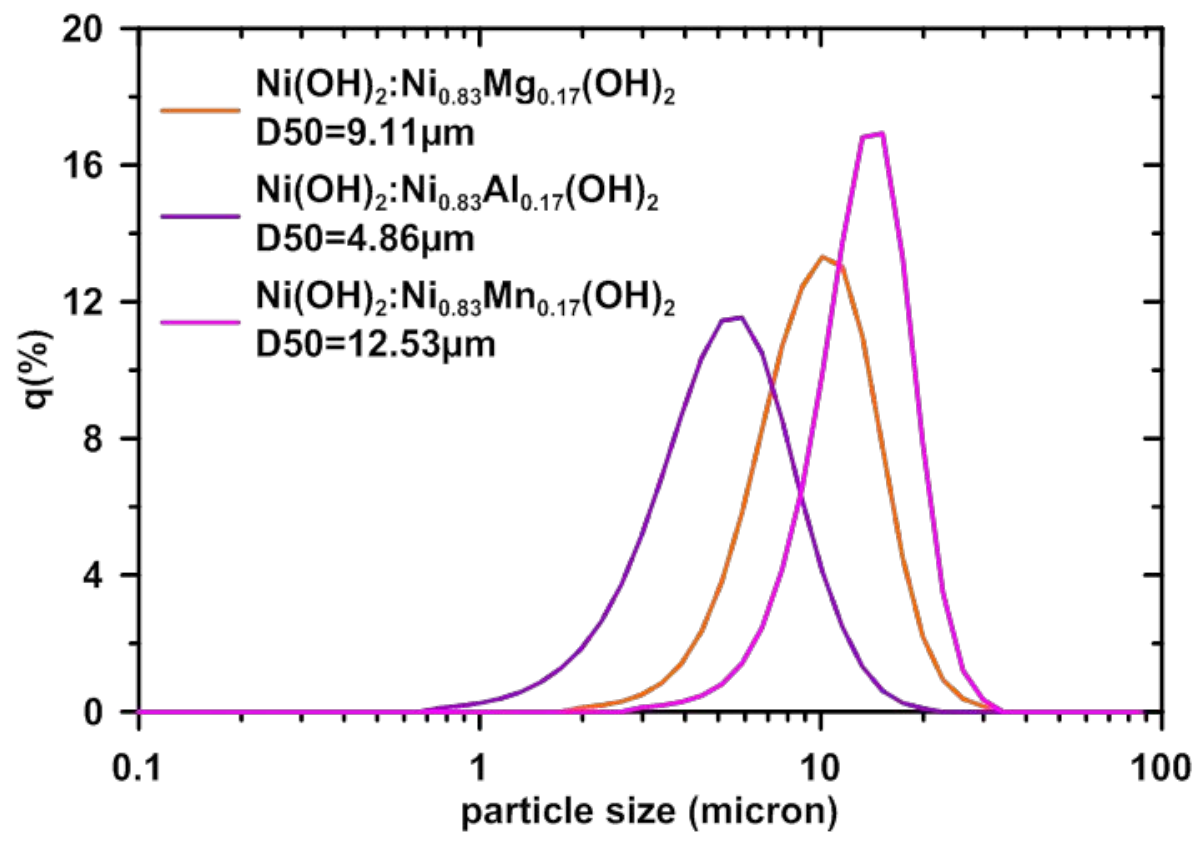


Figure S5. Cross-sectional SEM images of CS-LNO:NiMg83/17 in a) back scattering electron mode, b) secondary electron mode; cross-sectional SEM images of CS-LNO:NiA183/17 in c) back scattering electron mode, d) secondary electron mode; cross-sectional SEM images of CSLNO:NiMn83/17 in e) back scattering electron mode, f) secondary electron mode.

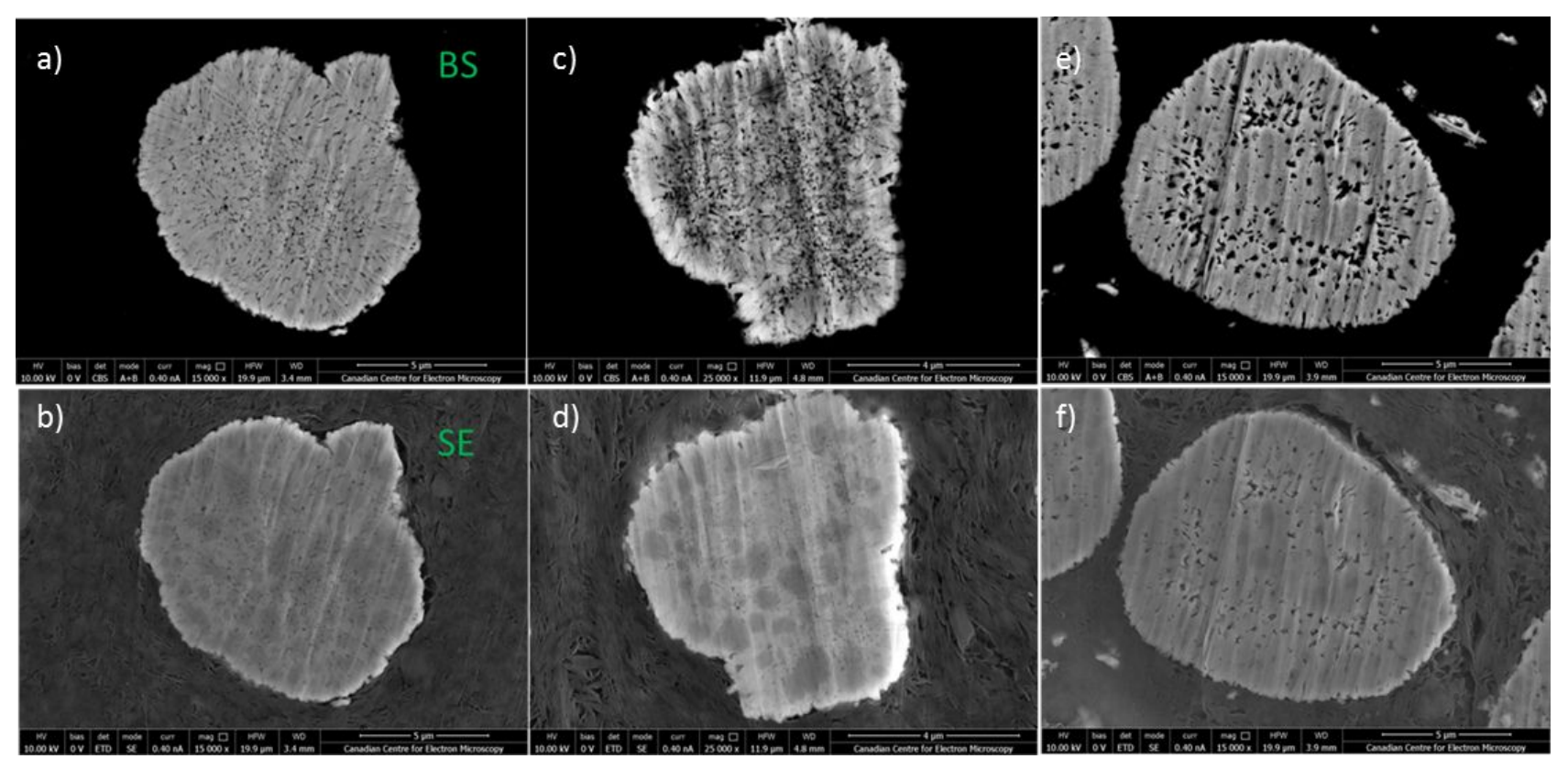


Figure S6. CS-LNO:NiAl83/17 in-situ XRD patterns at b) $18-21^{\circ}$, c) $36-40^{\circ}$, d) $43-51^{\circ}$, and e) $58-68^{\circ}$. a) Corresponding voltage curve from the in-situ half-cell charge and discharge at room temperature at around $\mathrm{C} / 100$. Each XRD scan from $17.5-68^{\circ}$ took around $2.85 \mathrm{~h}$. The first cycle was between 3.0-4.3 V, while the second charge was up to $4.7 \mathrm{~V}$.

a)

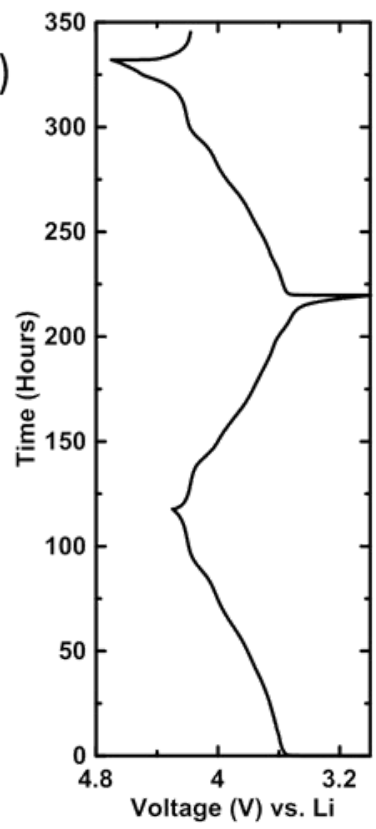

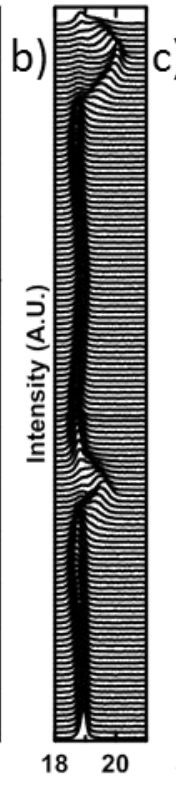
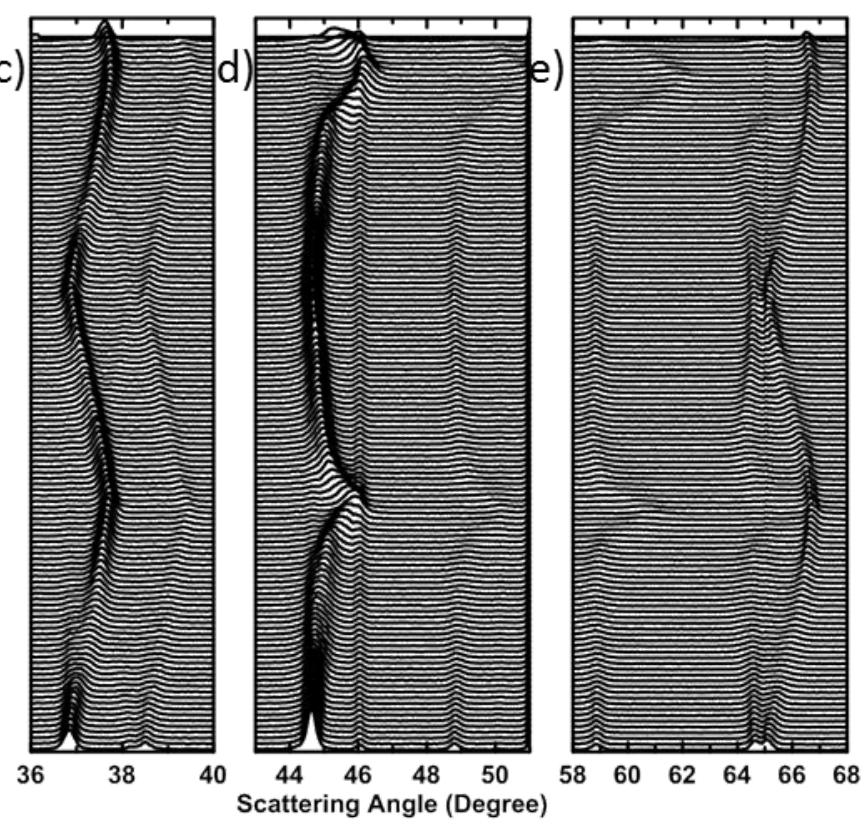
Figure S7. CS-LNO:NiA183/17 a) cell voltage, b) unit cell lattice constant a, c) unit cell lattice constant $\mathrm{c}$, and d) unit cell volume as a function of specific capacity.

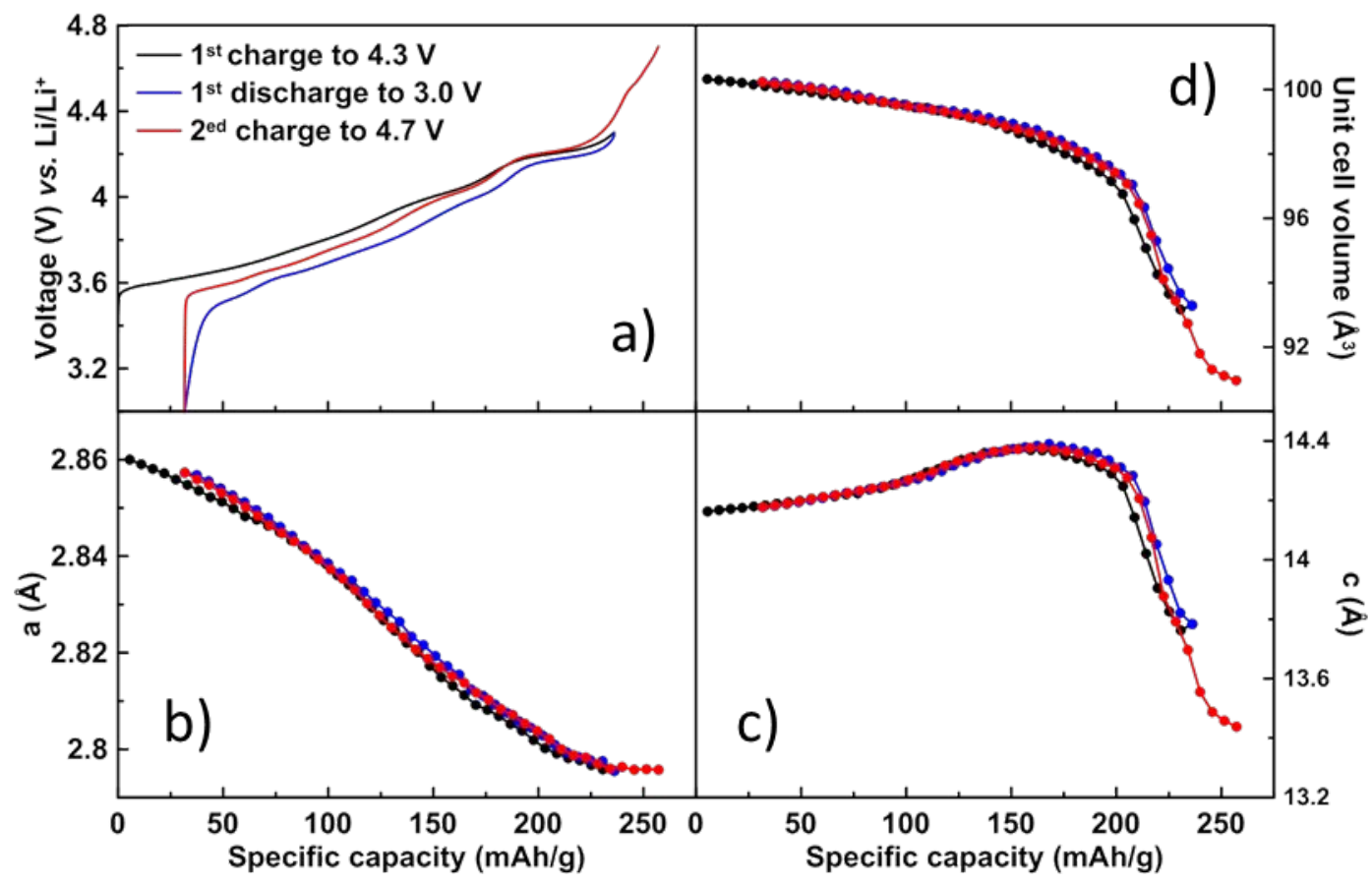


Figure S8. SEM images of CS-LNO:NiMn83/17 synthesized at $700^{\circ} \mathrm{C}$ with $\mathrm{Li} / \mathrm{TM}$ ratio of a) 0.98 , b) 1.00 , c) 1.02 , and d) 1.04 .

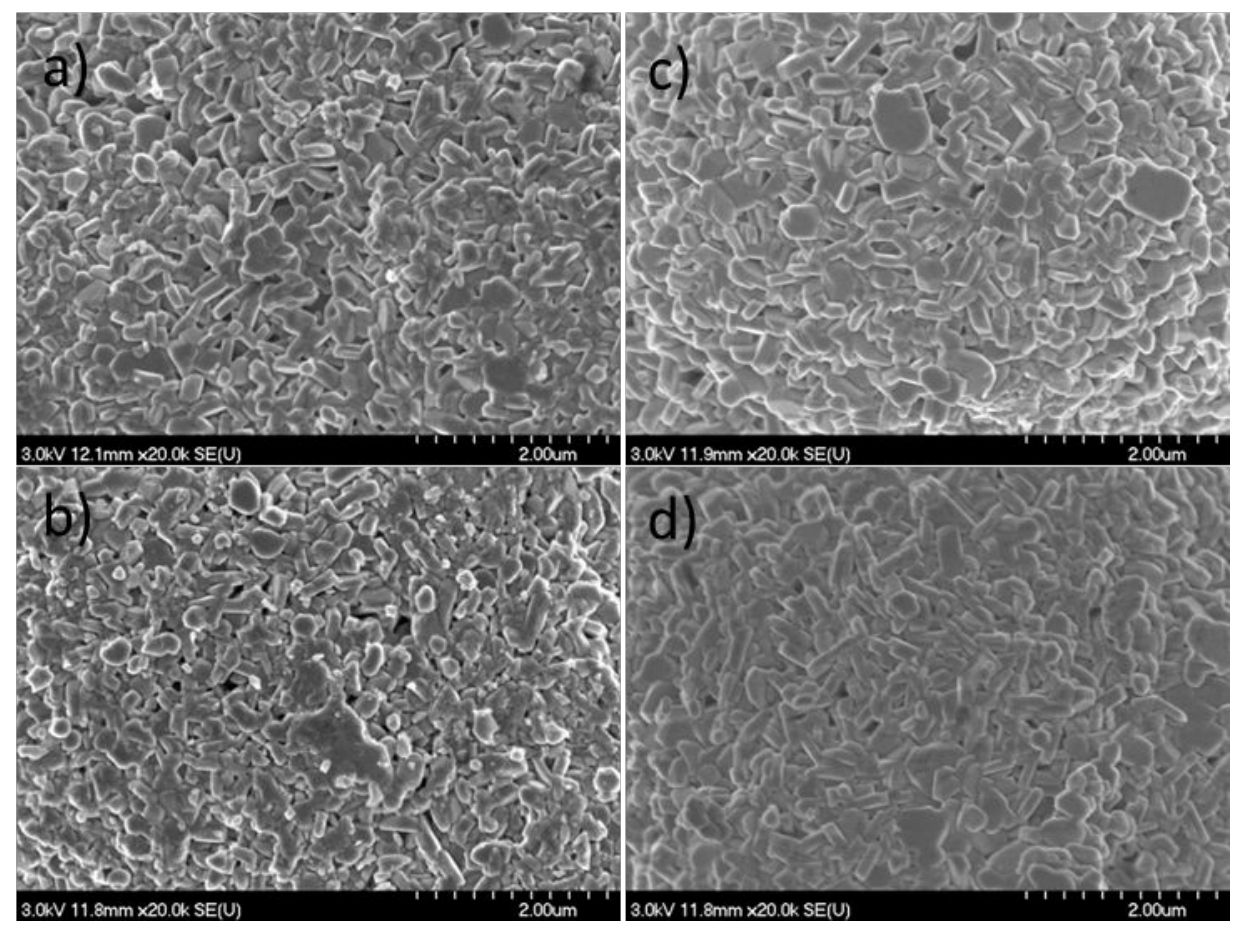


Figure S9. SEM images of $\mathrm{LiNi}_{0.95} \mathrm{Mn}_{0.05} \mathrm{O}_{2}$ synthesized at $735^{\circ} \mathrm{C}$ with $\mathrm{Li} / \mathrm{TM}$ ratio of a) 1.00, b) 1.04 , c) 1.08 , d) 1.12 , and e) 1.17 .
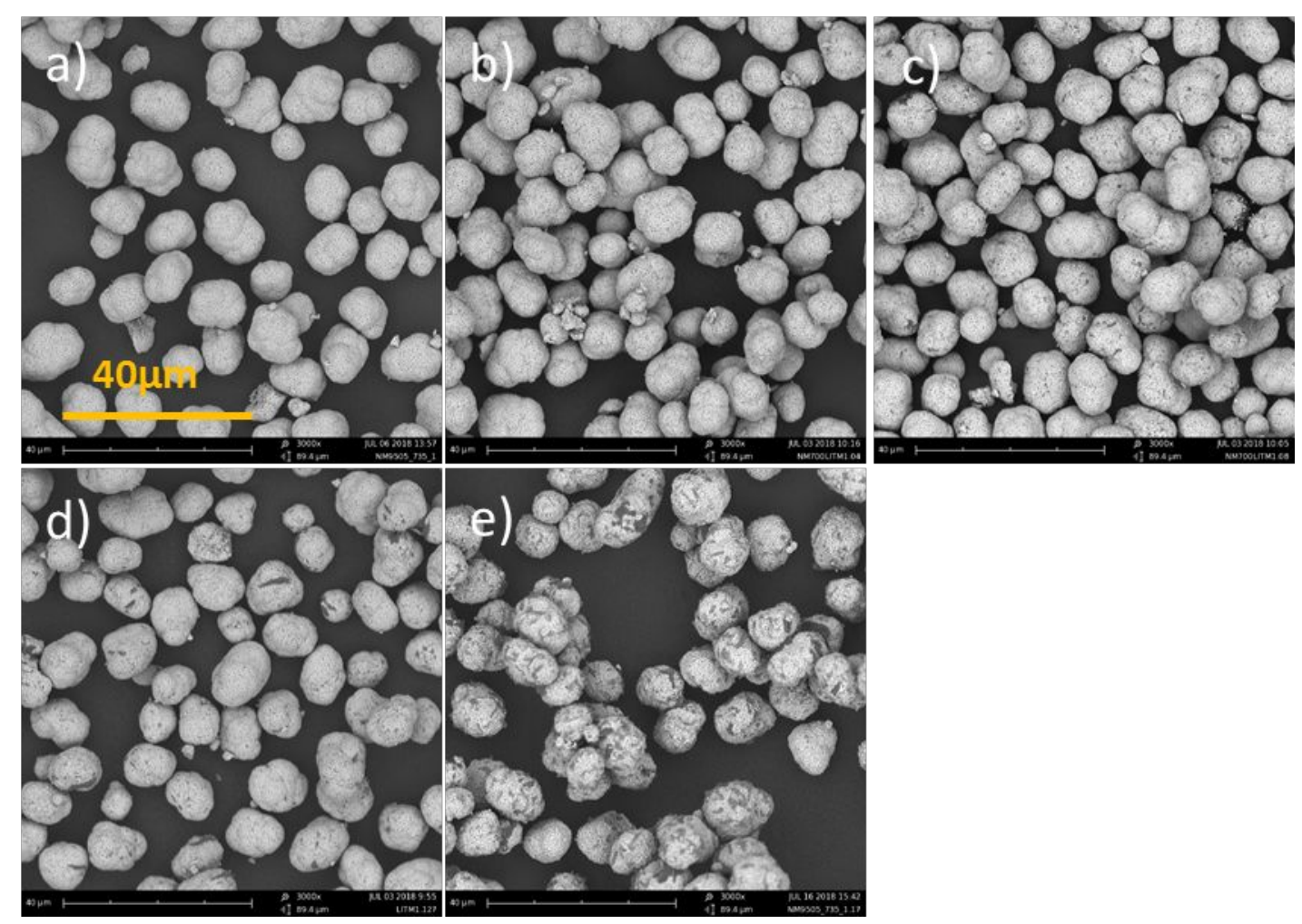